\title{
Gravitational Instability in a Ferromagnetic Fluid Saturated Porous Medium with Non-Classical Heat Conduction
}

\author{
Soya Mathew ${ }^{1}$, S. Maruthamanikandan ${ }^{2}$ and Smita S. Nagouda ${ }^{3}$ \\ ${ }^{I}$ Department of Science, BSF Institute of Technology, Bangalore-560 063, India \\ ${ }^{2,3}$ Department of Mathematics, Christ University, Bangalore-560 029, India
}

\begin{abstract}
The problem of Rayleigh-Bénard convection in a ferromagnetic fluid saturated porous medium with the Maxwell-Cattaneo law is studied by the method of small perturbation. Modified Darcy-Brinkman model is used to describe the fluid motion. The horizontal porous layer is cooled from the upper boundary, while an isothermal boundary condition is imposed at the lower boundary. The non-classical Maxwell-Cattaneo heat flux law involves a wave type heat transport and does not suffer from the physically unacceptable drawback of infinite heat propagation speed. The resulting eigenvalue problem is solved exactly for simplified boundary conditions and the thresholds for the marginal stability are determined. Some of the known cases are derived as special cases. The influence of porous, magnetic, and non-magnetic parameters on the onset of ferroconvection has been analyzed. It is found that the Bénard problem for a Maxwell-Cattaneo ferromagnetic fluid is always less stable than the classical ferroconvection problem. It is shown that the destabilizing influence of the Cattaneo number is not attenuated by that of magnetic forces and vice versa, and that the aspect ratio of the convection cells changes when the parameters involved in the study vary with the porous structure bringing out considerable influence.
\end{abstract}

Keywords - Darcy-Brinkman porous layer, Ferromagnetic fluid, Magnetization, Second sound, Stability.

\section{INTRODUCTION}

It is well known that ferromagnetic fluids represent a class of controllable liquids with interesting properties capable of making a substantial impact on technology. Ferromagnetic fluids have almost the same magnetic characteristics as a solid but in many respects behave as a liquid. Ferromagnetic fluids can be used to transfer heat inasmuch as heat and mass transport in such magnetic fluids can be controlled by means of an external magnetic field. Numerous applications can be associated with these fluids including novel energy conversion device, levitation devices and rotating seals (Bashtovoy et al. [1]). Finlayson [2] first explained how an external magnetic field imposed on a horizontal layer of ferromagnetic fluid with varying magnetic susceptibility due to a temperature gradient results in a non-uniform magnetic body force, which leads to thermomagnetic convection. This form of heat transfer can be useful for cases where conventional convection fails to provide adequate heat transfer, for instance, in miniature micro scale devices or under reduced gravity conditions.

Gupta and Gupta [3] investigated thermal instability in a layer of ferromagnetic fluid subject to coriolis force and permeated by a vertical magnetic field. It is substantiated that overstability cannot occur if the Prandtl number is greater than unity. Gotoh and Yamada [4] investigated the linear convective instability of a ferromagnetic fluid layer heated from below and confined between two horizontal ferromagnetic boundaries. The Galerkin technique is used and the Legendre polynomials are taken as the trial functions. It is shown that the magnetization of the boundaries and the nonlinearity of fluid magnetization reduce the critical Rayleigh number and the effects of magnetization and buoyancy forces are shown to compensate each other.

Blums [5] examined the possibility of having convection in ferromagnetic fluids as a result of magnetodiffusion of colloidal particles which gives rise to non-uniform magnetization. Stiles and Kagan [6] examined the thermoconvective instability of a horizontal layer of ferromagnetic fluid in a strong vertical magnetic field. Their work also questioned the satisfactory agreement claimed to exist between the experiments and the theoretical model of Finlayson [2] which has been generalized by them. Odenbach [7] investigated the convective flow generated by the interaction of a magnetic field gradient with a gradient in magnetization in a magnetic fluid. This gradient was caused by the diffusion of the magnetic particles in the field gradient. Aniss et al. [8] investigated the effect of a time-sinusoidal magnetic field on the onset of convection in a horizontal magnetic fluid layer heated from above. The Floquet theory is used to determine the convective threshold for free-free and rigid-rigid cases. The possibility to produce a competition between the harmonic and sub-harmonic modes at the onset of convection is discussed.

Abraham [9] investigated the $R B C$ problem in a micropolar ferromagnetic fluid layer in the presence of a vertical uniform magnetic field analytically. It is shown that the micropolar ferromagnetic fluid layer heated from below is more stable as compared with the classical Newtonian ferromagnetic fluid. The effect of radiative 
heat transfer on the onset of Rayleigh-Bénard convection in a horizontal layer of a ferromagnetic fluid has been studied by Maruthamanikandan [10] within the framework of linear stability analysis. Consideration is given to two asymptotic cases, viz., transparent and opaque layers of fluid. The critical values marking the onset of convection are obtained using the Rayleigh-Ritz technique.

Bajaj [11] considered thermosolutal convection in magnetic fluids in the presence of a vertical magnetic field and bifrequency vertical vibrations. The regions of parametric instability have been obtained using the Floquet theory. Maruthamanikandan [12] investigated the problem of gravitational instability in ferromagnetic fluids in the presence of internal heat generation, surface tension, and viscoelasticity. Ramanathan and Muchikel [13] investigated the effect of temperature-dependent viscosity on ferroconvective instability in a porous medium. It is found that the stationary mode of instability is preferred to oscillatory mode and that the effect of temperature-dependent viscosity has a destabilizing effect on the onset of convection.

Saravanan [14] made a theoretical investigation to study the influence of magnetic field on the onset of convection induced by centrifugal acceleration in a magnetic fluid filled porous medium. The layer is assumed to exhibit anisotropy in mechanical as well as thermal sense. Numerical solutions are obtained using the Galerkin method. It is found that the magnetic field has a destabilizing effect and can be suitably adjusted depending on the anisotropy parameters to enhance convection. The effect of anisotropies of magnetic fluid filled porous media is shown to be qualitatively different from that of ordinary fluid filled porous media.

Singh and Bajaj [15] investigated numerically the effect of frequency of modulation, applied magnetic field, and Prandtl number on the onset of a periodic flow in a ferromagnetic fluid layer using the Floquet theory. Some theoretical results have been obtained to discuss the limiting behavior of the underlying instability with the temperature modulation. Depending upon the parameters, the flow patterns at the onset of instability have been found to consist of time-periodically oscillating vertical magnetoconvective rolls. Singh and Bajaj [16] considered the effect of time-periodic modulation in temperatures on the onset of ferroconvection with rigid boundaries. It is found that, under modulation, subcritical instabilities are found to occur in the form of subharmonic response. Also, the onset of instability in a ferromagnetic fluid layer is found to heavily depend upon the frequency of modulation when it is driven solely by the magnetic forces alone, the effect being the greatest for the low frequency modulation and negligible for the case of high frequency modulation.

In view of the fact that heat transfer can be greatly enhanced due to thermomagnetic convection, the ferroconvection problems offer fascinating applications including cooling with motors, loudspeakers and transmission lines.

The study of convection in a fluid saturated porous medium is of considerable interest due to its applications to chemical engineering, geothermal activities, oil recovery techniques and biological processes. Over the past couple of decades, a great deal of effort has been invested in studying natural convection in a fluid-saturated porous medium with and without uniformly distributed heat source keeping in mind the applications to nuclear reactor safety and geothermal reservoir engineering. An illuminating and detailed information on convective instability in porous media is available in the books of Ingham and Pop [17], and Nield and Bejan [18], and Vafai [19]. It is worth noting that porous medium convection has been given much attention because allowance of porous medium significantly simplifies the description of an average hydrodynamic flow and enables us to consider realistic boundary conditions for the velocity.

The propagation of thermal waves is sometimes referred to as second sound effect. The classical energy equation allows for an infinite speed for the propagation of heat, which is physically unacceptable. The energy equation to be considered in the present work is effectively a damped wave equation and is therefore hyperbolic rather than parabolic. The knowledge of second sound has provided a rich source of information for the study and understanding of the superfluid state. Second sound is not in any sense a sound wave, but a temperature or entropy wave. In ordinary or first sound, pressure and density variations propagate with very small accompanying variations in temperature; in second sound, temperature variations propagate with no appreciable variations in density or pressure. Recently, it has been realized that this is not just a low temperature phenomenon, but has important applications in such fields as skin burns, phase changes, biological materials, and in nanofluids (Straughan [20]).

Straughan and Franchi [21] investigated the effect of thermal waves upon the onset of convective instability of a Newtonian fluid confined in a horizontal layer of finite thickness. Stress-free boundaries have been considered. It is found that convection is possible in both heated above and below cases, the Benard problem for a Maxwell-Cattaneo fluid is always less stable than the classical one and overstability only occurs in the heated below case.

Lebon and Cloot [22] examined the effects resulting from the substitution of the classical Fourier law of heat conduction by the Maxwell-Cattaneo law in Bénard's and Marangoni's problems. Considering only infinitesimally small perturbations, it is shown that when buoyancy is the single factor of instability, no stationary convection can develop in a fluid layer heated from above, but oscillatory convection is possible. It is found that in Maxwell-Cattaneo fluid, oscillatory convection does not play an important practical role. 
Straughan [20] investigated the problem of thermal convection for a layer of fluid when the heat flux law of Cattaneo is adopted. The boundary conditions are taken to be rigid-rigid and isothermal. It is shown that for small Cattaneo number the critical Rayleigh number initially increases from its classical value until a critical value of the Cattaneo number is reached. For Cattaneo numbers greater than this critical value a notable Hopf bifurcation is observed with convection occurring at lower Rayleigh numbers and by oscillatory rather than stationary convection. It is also found that the aspect ratio of the convection cells likewise changes.

Smita and Pranesh [23] studied the problem of the onset of Rayleigh-Bénard convection in a second order Colemann-Noll fluid by replacing the classical Fourier heat flux law with non-classical Maxwell-Cattaneo law. The Galerkin method is employed to determine the critical values. The eigenvalue problem is solved using the general boundary conditions on velocity and third type of boundary conditions on temperature. It is found that the classical Fourier heat flux law overpredicts the critical Rayleigh number compared to that predicted by the non-classical law and that the results are noteworthy at short times. Moreover, overstability is the preferred mode of convection.

The problem of control of convection is of relevance and interest in innumerable ferromagnetic fluid applications and is also mathematically quite challenging. The problem of Rayleigh-Bénard convection in a ferromagnetic fluid saturated porous medium has been extensively studied. However, attention has not been paid to the effect of second sound on Rayleigh-Bénard convection in a horizontal porous layer of a ferromagnetic fluid. It is with this motivation that we study, in this paper, the problem of natural convection in a ferromagnetic fluid saturated porous layer subject to non-classical heat conduction with emphasis on how the stability criterion for the onset of ferroconvection would be modified in the presence of both the porous matrix and second sound. While the effect of a variety of non-uniform basic temperature gradients on the onset of ferroconvection has been studied intensely [12], we have seen no work dealing with the effect of thermal waves upon the onset of ferroconvective instability in a porous layer. Under these circumstances, the present paper is devoted to studying qualitatively the effect of propagation of thermal waves on the onset of ferroconvection in a horizontal porous layer. The linear stability analysis is based on the normal mode technique. The modified Darcy-Brinkman law is used to model the momentum equation and we allow for a thermal wave of finite speed by adopting the heat flux model of Cattaneo.

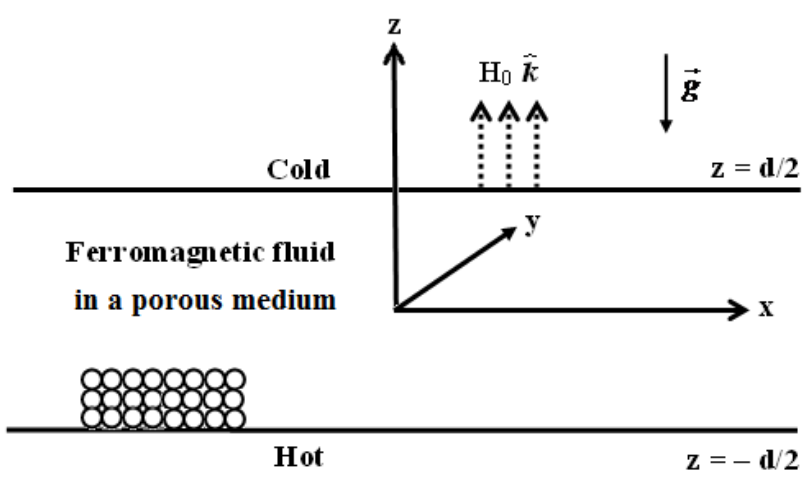

Fig. 1 Configuration of the problem.

\section{Mathematical Formulation}

We consider a Boussinesq ferromagnetic fluid saturated sparsely distributed porous layer confined between two infinite horizontal surfaces with height ' $d$ '. A vertical downward gravity force acts on the fluid together with a uniform, vertical magnetic field $\vec{H}_{\mathrm{O}}$. A Cartesian frame of reference is chosen with the origin in the lower boundary and the $z$-axis vertically upwards. The lower surface at $z=0$ and upper surface at $z=d$ are maintained at constant temperatures $T_{1}$ and $T_{\mathrm{O}}$. The Boussinesq approximation is invoked to account for the effect of density variation. The governing equations describing flow in an incompressible ferromagnetic fluid are

$$
\begin{aligned}
& \nabla \cdot \vec{q}=0 \\
& \rho_{\mathrm{O}}\left[\frac{1}{\varepsilon} \frac{\partial \vec{q}}{\partial t}+\frac{1}{\varepsilon^{2}}(\vec{q} \cdot \nabla) \vec{q}\right]=-\nabla p+\rho \vec{g}-\frac{\mu_{f}}{k} \vec{q}+\bar{\mu}_{f} \nabla^{2} \vec{q}+\nabla \cdot(\vec{H} \vec{B})
\end{aligned}
$$




$$
\begin{gathered}
\varepsilon\left[\rho_{\mathrm{O}} \bar{C}_{V, H}-\mu_{\mathrm{O}} \vec{H} \cdot\left(\frac{\partial \vec{M}}{\partial T}\right)_{V, H}\right]\left[\frac{\partial T}{\partial t}+\vec{q} \cdot \nabla T\right]+(1-\varepsilon)\left(\rho_{\mathrm{o}} \bar{C}\right)_{S} \frac{\partial T}{\partial t} \\
+\mu_{\mathrm{o}} T\left(\frac{\partial \vec{M}}{\partial T}\right)_{V, H} \cdot\left[\frac{\partial \vec{H}}{\partial t}+(\vec{q} \cdot \nabla) \vec{H}\right]=-\nabla \cdot \vec{Q} \\
\tau\left[\frac{\partial \vec{Q}}{\partial t}+(\vec{q} \cdot \nabla) \vec{Q}+\vec{\omega} \times \vec{Q}\right]=-\vec{Q}-k_{1} \nabla T \\
\rho=\rho_{\mathrm{o}}\left[1-\alpha\left(T-T_{a}\right)\right]
\end{gathered}
$$

where $\vec{q}=(u, v, w)$ is the fluid velocity, $\varepsilon$ the porosity of the porous medium, $t$ the time, $p$ the pressure, $\rho$ the fluid density, $\vec{g}$ the acceleration due to gravity, $\mu_{f}$ the dynamic viscosity, $\bar{\mu}_{f}$ the effective viscosity, $k$ the permeability of the porous medium, $\vec{H}$ the magnetic field, $\vec{B}$ the magnetic induction, $\rho_{\mathrm{O}}$ a reference density, $\bar{C}$ the specific heat, $\bar{C}_{V, H}$ the specific heat at constant volume and constant magnetic field, $\mu_{\mathrm{o}}$ the magnetic permeability, $T$ the temperature, $\vec{M}$ the magnetization, $\tau$ the constant relaxation time, $k_{1}$ the thermal conductivity, $\alpha$ the coefficient of thermal expansion, $T_{a}$ the arithmetic mean of boundary temperatures, $\vec{Q}$ the heat flux vector, $\vec{\omega}=\frac{1}{2}(\nabla \times \vec{q})$ and the subscript $s$ represents the solid.

Maxwell's equations simplified for a non-conducting fluid with no displacement current take the form

$\nabla \cdot \vec{B}=0, \quad \nabla \times \vec{H}=\overrightarrow{0}$

$\vec{B}=\mu_{\mathrm{o}}(\vec{H}+\vec{M})$.

Since the magnetization $\vec{M}$ is aligned with the magnetic field and is a function of temperature and magnetic field, we may write

$\vec{M}=\frac{\vec{H}}{H} M(H, T)$.

The magnetic equation of state is linearized about the magnetic field $\vec{H}_{\mathrm{O}}$ and the reference temperature $T_{a}$ to become

$M=M_{\mathrm{o}}+\chi\left(H-H_{\mathrm{o}}\right)-K\left(T-T_{a}\right)$

where $\chi$ is the magnetic susceptibility and $K$ is the pyromagnetic coefficient.

\section{Basic State}

The basic state is quiescent and is given by

$\left.\begin{array}{l}\vec{q}=\vec{q}_{b}=(0,0,0), \quad \rho=\rho_{b}(z), \quad T=T_{b}(z), \quad p=p_{b}(z), \\ \vec{H}=\vec{H}_{b}=\left(0,0, H_{b}(z)\right), \vec{M}=\vec{M}_{b}=\left(0,0, M_{b}(z)\right), \\ \vec{B}=\vec{B}_{b}=\left(0,0, B_{b}(z)\right), \quad \vec{Q}=\vec{Q}_{b}=\left(0,0, k_{1} \beta\right)\end{array}\right\}$

where the subscript $b$ denotes the basic state and $\beta=\frac{T_{1}-T_{\mathrm{O}}}{d}$. The quiescent basic state has a solution in the form

$$
\begin{aligned}
& T_{b}(z)=T_{a}-\beta z \\
& \rho_{b}=\rho_{\mathrm{o}}[1+\alpha \beta z] \\
& H_{b}(z)=H_{\mathrm{O}}-\frac{K \beta z}{1+\chi} \\
& M_{b}(z)=M_{\mathrm{O}}+\frac{K \beta z}{1+\chi}
\end{aligned}
$$


where $H_{\mathrm{O}}$ is the uniform magnetic field and $M_{\mathrm{O}}$ is the reference magnetization. The analysis is restricted to physical situations in which the magnetization induced by temperature variations is small compared to that induced by the external magnetic field.

\section{Perturbed State}

Since we are interested in the stability of the basic state, we superimpose infinitesimally small perturbations on the basic state according to

$$
\left.\begin{array}{l}
\vec{q}=\vec{q}_{b}+\vec{q}^{\prime}=\left(u^{\prime}, v^{\prime}, w^{\prime}\right), \quad p=p_{b}+p^{\prime}, \quad \rho=\rho_{b}+\rho^{\prime}, \\
T=T_{b}+T^{\prime}, \quad \vec{H}=\vec{H}_{b}+\vec{H}^{\prime}, \quad \vec{M}=\vec{M}_{b}+\vec{M}^{\prime}, \\
\vec{B}=\vec{B}_{b}+\vec{B}^{\prime}, \quad \phi=\phi_{b}+\phi^{\prime}, \quad \vec{Q}=\vec{Q}_{b}+\vec{Q}^{\prime}
\end{array}\right\}
$$

where primes denote perturbed quantities and $\vec{H}=\nabla \phi^{\prime}$ with $\phi^{\prime}$ being the magnetic potential.

Following the classical procedure of linear stability analysis, the linearized equations governing small perturbations turn out to be

$$
\begin{aligned}
& \rho_{\mathrm{O}} \frac{\partial}{\partial t}\left(\nabla^{2} w^{\prime}\right)=\alpha \rho_{\mathrm{O}} g \nabla_{1}^{2} T^{\prime}-\frac{\mu_{f}}{k} \nabla^{2} w^{\prime}+\bar{\mu}_{f} \nabla^{4} w^{\prime}+\frac{\mu_{\mathrm{O}} K^{2} \beta}{1+\chi} \nabla_{1}^{2} T^{\prime}-\mu_{\mathrm{O}} K \beta \frac{\partial}{\partial z}\left(\nabla_{1}^{2} \phi^{\prime}\right) \\
& \left(1+\tau \frac{\partial}{\partial t}\right)\left[\left(\rho_{\mathrm{O}} \bar{C}\right)_{1} \frac{\partial T^{\prime}}{\partial t}-\mu_{\mathrm{O}} K T_{a} \frac{\partial}{\partial t}\left(\frac{\partial \phi^{\prime}}{\partial z}\right)-\left\{\left(\rho_{\mathrm{O}} \bar{C}\right)_{2}-\frac{\mu_{\mathrm{O}} K^{2} T_{a}}{1+\chi}\right\} \beta w^{\prime}\right]=k_{1} \nabla^{2} T^{\prime}-\frac{\tau k_{1} \beta}{2} \nabla^{2} w^{\prime} \\
& \left(1+\frac{M_{\mathrm{O}}}{H_{\mathrm{O}}}\right) \nabla_{1}^{2} \phi^{\prime}+(1+\chi) \frac{\partial^{2} \phi^{\prime}}{\partial z^{2}}-K \frac{\partial T^{\prime}}{\partial z}=0
\end{aligned}
$$

where $\left(\rho_{\mathrm{o}} \bar{C}\right)_{1}=\varepsilon \rho_{\mathrm{O}} \bar{C}_{V, H}+\varepsilon \mu_{\mathrm{O}} K H_{\mathrm{O}}+(1-\varepsilon)\left(\rho_{\mathrm{o}} \bar{C}\right)_{S}$ and $\left(\rho_{\mathrm{o}} \bar{C}\right)_{2}=\varepsilon \rho_{\mathrm{O}} \bar{C}_{V, H}+\varepsilon \mu_{\mathrm{o}} K H_{\mathrm{O}}$. We use the normal mode solution for the dependent variables in the form

$$
\left[\begin{array}{c}
w^{\prime} \\
T^{\prime} \\
\phi^{\prime}
\end{array}\right]=\left[\begin{array}{l}
W(z) \\
\Theta(z) \\
\Phi(z)
\end{array}\right] e^{i(l x+m y)+\sigma t}
$$

where $l$ and $m$ are the dimensionless wavenumbers in the $x$ and $y$ directions respectively and $\sigma$ is the growth rate. Substitution of (19) into Eqs. (16) - (18) yields

$$
\begin{aligned}
& \rho_{\mathrm{O}} \sigma\left(D^{2}-k_{h}^{2}\right) W=-\alpha \rho_{\mathrm{o}} g k_{h}^{2} \Theta-\frac{\mu_{f}}{k}\left(D^{2}-k_{h}^{2}\right) W+\bar{\mu}_{f}\left(D^{2}-k_{h}^{2}\right)^{2} W-\frac{\mu_{\mathrm{O}} K^{2} \beta}{1+\chi} k_{h}^{2} \Theta+\mu_{\mathrm{O}} K \beta k_{h}^{2} D \Phi \\
& (1+\tau \sigma)\left[\left(\rho_{\mathrm{O}} \bar{C}\right)_{1} \sigma \Theta-\mu_{\mathrm{O}} K T_{a} \sigma D \Phi-\left\{\left(\rho_{\mathrm{O}} \bar{C}\right)_{2}-\frac{\mu_{\mathrm{O}} K^{2} T_{a}}{1+\chi}\right\} \beta W\right]=k_{1}\left(D^{2}-k_{h}^{2}\right) \Theta-\frac{\tau k_{1} \beta}{2}\left(D^{2}-k_{h}^{2}\right) W \\
& (1+\chi) D^{2} \Phi-\left(1+\frac{M_{\mathrm{O}}}{H_{\mathrm{O}}}\right) k_{h}^{2} \Phi-K D \Theta=0
\end{aligned}
$$

where $D=\frac{d}{d z}$ and $k_{h}^{2}=l^{2}+m^{2}$ is the overall horizontal wavenumber of the convective disturbance. Non-dimensionalizing equations (20), (21) and (22) using the transformations

$$
W^{*}=\frac{W d}{\kappa}, \Phi^{*}=\frac{\Phi(1+\chi)}{K \beta d^{2}}, \quad \sigma^{*}=\frac{\sigma d^{2}}{\kappa}, \Theta^{*}=\frac{\Theta}{\beta d}, z^{*}=\frac{z}{d}, a=k_{h} d, \quad \kappa=\frac{k_{1}}{\left(\rho_{\mathrm{o}} \bar{C}\right)_{2}}
$$

we obtain

$$
\begin{aligned}
& \frac{\sigma}{P r}\left(D^{2}-a^{2}\right) W=-(R+N) a^{2} \Theta-D a^{-1}\left(D^{2}-a^{2}\right) W+\Lambda\left(D^{2}-a^{2}\right)^{2} W+N a^{2} D \Phi \\
& (1+2 C \sigma) \lambda \sigma \Theta=(1+2 C \sigma) W+\left(D^{2}-a^{2}\right) \Theta-C\left(D^{2}-a^{2}\right) W \\
& D^{2} \Phi-M_{3} a^{2} \Phi-D \Theta=0
\end{aligned}
$$


where $\operatorname{Pr}=\frac{\mu_{f}}{\rho_{\mathrm{o}} \kappa}$ is the Prandtl number, $D a^{-1}=\frac{d^{2}}{k}$ is the inverse Darcy number, $\Lambda=\frac{\bar{\mu}_{f}}{\mu_{f}}$ is the Brinkman number, $R=\frac{\alpha \rho_{0} g \beta d^{4}}{\mu_{f} \kappa}$ is the thermal Rayleigh number, $N=\frac{\mu_{0} K^{2} \beta^{2} d^{4}}{\mu_{f}(1+\chi) \kappa}$ is the magnetic Rayleigh number, $C=\frac{\tau \kappa}{2 d^{2}}$ is the Cattaneo number and $\lambda=\frac{\left(\rho_{\mathrm{O}} \bar{C}\right)_{1}}{\left(\rho_{\mathrm{O}} \bar{C}\right)_{2}}$. The usual boundary conditions apply and for two free, isothermal surfaces they are (Chandrasekhkar [24])

$W=D^{2} W=\Theta=D \Phi=0$ at $z= \pm 1 / 2$

It should be noted that the boundary condition $D \Phi=0$ is indicative of the fact that the magnetic susceptibility is very large at the boundaries. Finlayson [2] used this type of boundary condition in order to obtain exact solution to the ferroconvective instability problem with free-free, isothermal boundaries. The derivation of the general boundary conditions on the magnetic potential is given in the work of Maruthamanikandan [12]. Noting that the principle of exchange of stabilities is valid $[2,22,25]$, we arrive at the following stability equations

$$
\begin{aligned}
& \Lambda\left(D^{2}-a^{2}\right)^{2} W-D a^{-1}\left(D^{2}-a^{2}\right) W-(R+N) a^{2} \Theta+N a^{2} D \Phi=0 \\
& C\left(D^{2}-a^{2}\right) W-\left(D^{2}-a^{2}\right) \Theta-W=0 \\
& \left(D^{2}-M_{3} a^{2}\right) \Phi-D \Theta=0 .
\end{aligned}
$$

\section{EXACT SOLUTION}

Because the linear eigenvalue system (28) - (30) has constant coefficients, it has a general solution with an exponential dependence on $z$. Equations (28), (29) and (30) together with boundary conditions (27) constitute an eigenvalue problem with $R$ being the eigenvalue. Let us assume the solution in the following form so that they satisfy the boundary conditions

$$
W=A_{1} \cos \pi z, \Theta=A_{2} \cos \pi z, \Phi=\frac{A_{3}}{\pi} \sin \pi z
$$

where $A_{1}, A_{2}$ and $A_{3}$ are constants. The condition for the existence of a non-trivial eigenvalue leads to the following expression for $R$

$$
R=\frac{\left[D a^{-1}\left(\pi^{2}+a^{2}\right)+\Lambda\left(\pi^{2}+a^{2}\right)^{2}\right]\left(\pi^{2}+a^{2}\right)}{a^{2}\left[1+C\left(\pi^{2}+a^{2}\right)\right]}-\frac{N M_{3} a^{2}}{\left(M_{3} a^{2}+\pi^{2}\right)} .
$$

Before developing the consequences of (32), we mention a couple of limiting cases that can be derived from (32). In the limiting case of $C=0, D a \rightarrow \infty$ and $\Lambda=1$, one recovers the result of Finlayson [2] and the associated Rayleigh number $R$ is given by

$$
R=\frac{\left(\pi^{2}+a^{2}\right)^{3}}{a^{2}}-\frac{N M_{3} a^{2}}{\left(M_{3} a^{2}+\pi^{2}\right)} .
$$

When $C=0$, one obtains the Rayleigh number expression for Darcy-Brinkman ferroconvection problem [25] and the same is given by

$$
R=\frac{\left[D a^{-1}\left(\pi^{2}+a^{2}\right)+\Lambda\left(\pi^{2}+a^{2}\right)^{2}\right]\left(\pi^{2}+a^{2}\right)}{a^{2}}-\frac{N M_{3} a^{2}}{\left(M_{3} a^{2}+\pi^{2}\right)} .
$$

In the limiting case of $N=0, D a \rightarrow \infty$ and $\Lambda=1$, one recovers the result of Lebon and Cloot [22] and the corresponding Rayleigh number $R$ is given by 


$$
R=\frac{\left(\pi^{2}+a^{2}\right)^{3}}{a^{2}\left[1+C\left(\pi^{2}+a^{2}\right)\right]}
$$

In this case $R$ assumes its minimum value at the critical wavenumber $a_{c}^{2}=\frac{\sqrt{1+C \pi^{2}\left(1+C \pi^{2}\right)}-1}{C}$. When $N=C=0, D a \rightarrow \infty$ and $\Lambda=1$, we obtain

$$
R=\frac{\left(\pi^{2}+a^{2}\right)^{3}}{a^{2}}
$$

which is the well-known expression concerning the problem of Rayleigh-Bénard convection in a Newtonian Fourier fluid in the absence of porous medium [24]. We find that $R$ assumes its minimum value $R_{c}=27 \pi^{4} / 4$ at $a_{C}=\pi / \sqrt{2}$. Moreover, in the limiting case of $N=C=0, D a \rightarrow 1$ and $\Lambda=0$, one recovers the classical result pertaining to Darcy porous medium convection in a Newtonian Fourier fluid [18] and the corresponding Rayleigh number $R$ is given by

$$
R=\frac{\left(\pi^{2}+a^{2}\right)^{2}}{a^{2}} \text {. }
$$

We find that $R$ assumes its minimum value $R_{c}=4 \pi^{2}$ at $a_{c}=\pi$.

\section{RESULTS AND DISCUSSION}

The problem of convective instability driven by a coupling of thermal, porous and magnetic effects in an initially quiescent ferromagnetic fluid saturated porous layer is investigated analytically by the method of small perturbation. It should be noted that the heat is transferred purely by conduction in the quiescent state and by both conduction and convection in the steady convective state. It is also of interest to note that for nondissipative flows there is an alternative variational approach to stability which relies on determining whether or not the energy of the flow is a minimum at equilibrium [26]. It should be remarked that the use of realistic flow boundary conditions does not qualitatively but quantitatively change the critical values. Similarly the use of realistic boundary conditions on the magnetic potential is of only very limited impact on the stability of the system. It is well known that rigid-rigid boundaries offer most stabilizing effect against the fluid motion and the least suppression is offered by free-free boundaries [24].

It is known from thermodynamics that the relaxation time and consequently the parameter $C$ are positive quantities. It has been established that oscillatory convection occurs only for $C$ above a threshold value and since the $C$ values encountered with laboratory fluids appear well below this threshold it is advantageous to concentrate on stationary convection. The thermal Rayleigh number $R$, characterizing the stability of the system, is calculated as a function of the magnetic and non-magnetic parameters. The role of various magnetic and non-magnetic properties and their mutual interplay for the instability is examined. The values of the parameters arising in the study are fairly standard and are experimentally relevant $[12,18,22]$. Neutral stability curves in the $(R, a)$ plane are plotted for different values of the governing parameters. The coordinates of the lowest point on these curves designate the critical values of $R$ and $a$. The fixed values of $C, M_{3}, D a^{-1}$ and $\Lambda$ are taken to be $0.001,1,5$ and 3 respectively.

The dependence of critical thermal Rayleigh number $R_{c}$ on the magnetic Rayleigh number $N$ for different values of $C$ and for fixed values of $M_{3}, D a^{-1}$ and $\Lambda$ is sketched in Fig. 2. The magnetic Rayleigh number $N$ is a ratio of the magnetic to dissipative forces. It is found that $R_{c}$ decreases monotonically with an increase in both $N$ and $C$ indicating that both $N$ and $C$ cause ferroconvection to occur at lower value of $R_{C}$. As a result, the two physical mechanisms, viz., second sound and magnetic mechanism have destabilising effect on the system. In other words, the presence of magnetic field and second sound facilitates heat transfer more effectively and hence hastens the onset of Darcy-Brinkman ferroconvection. It is interesting to note that the effect of $C$ is to reinforce the destabilising effect of $N$ and vice versa. The reason for the destabilizing effect of 
$C$ is that the energy equation considered is effectively a damped wave equation and is, therefore, hyperbolic rather than parabolic. As for the destabilizing effect of $N$, since the external magnetic field induces magnetization in the ferromagnetic fluid and magnetization is a function of both magnetic field and temperature, the applied temperature causes a spatial variation in the magnetization, which is the driving force causing the convection. For small temperature gradients the magnetic forces induced by the temperature gradient cannot overcome the viscous and thermal dissipation and the layer remains quiescent. When the temperature gradient is sufficiently large, fluid motion occurs. It is established that the magnetic mechanism predominates over the buoyancy mechanism in thin fluid layers [2].

In Fig. 3, the variation of critical thermal Rayleigh number $R_{c}$ with magnetic Rayleigh number $N$ for different values of the magnetization parameter $M_{3}$ and for fixed values of $C, D a^{-1}$ and $\Lambda$ is shown. The parameter $M_{3}$ represents the departure of the magnetic equation of state from linearity. We see that the effect of increasing $M_{3}$ is to decrease $R_{c}$ monotonically. Thus the threshold of ferroconvection in a porous layer with second sound is hastened as the magnetic equation of state becomes more and more nonlinear. Also, the destabilizing effect of $M_{3}$ is almost insignificant when $N$ is small.

The effect of porous parameters $D a^{-1}$ and $\Lambda$ on the onset of ferroconvection is depicted in Figs. 4 and 5. It is clear that the onset of ferroconvection is delayed when both $D a^{-1}$ and $\Lambda$ are increased. This may be attributed to the fact that increase in $D a^{-1}$ amounts to decreasing the permeability of the porous medium which in turn retards the fluid flow. On the other hand, it is advantageous to note that the Brinkman model rests on an effective viscosity $\bar{\mu}_{f}$ different from the fluid viscosity $\mu_{f}$ designated through the Brinkman number $\Lambda$. So increasing $\Lambda$ amounts to increasing the viscous effect which in turn retards the fluid flow. Therefore more heating is required to set off ferroconvection in the presence of Darcy-Brinkman porous medium. It is striking to note that the ferrofluid layer is destabilized slightly through the magnetic mechanism when both $D a^{-1}$ and $\Lambda$ are large.

The effect of $N, M_{3}, C, D a^{-1}$ and $\Lambda$ on the size of the convection cell is displayed in Figs. 6 through 9. The dimensionless wavenumber $a$ is the characteristic of the cell shape and size. A close examination of these figures reveals that $a_{c}$ increases with an increase in $N, C$ and $D a^{-1}$, and decreases with increasing $M_{3}$ and $\Lambda$ implying that the effect of $N, C$ and $D a^{-1}$ is to contract the convection cell size and that of $M_{3}$ and $\Lambda$ is to enlarge the size of convection cell. Thus all the parameters of the study have a say on the aspect ratio of the convection cell with the effect of $D a^{-1}$ and $\Lambda$ being most significant. In the light of the Cattaneo law being a possible mechanism for heat transfer in ferrofluids, we believe that the results of the study at hand might be exploited in heat transfer devices wherein magnetic fluids play a prominent role and that the results presented here may have some bearing on a suitable laboratory experiment.

\section{FIGURES}

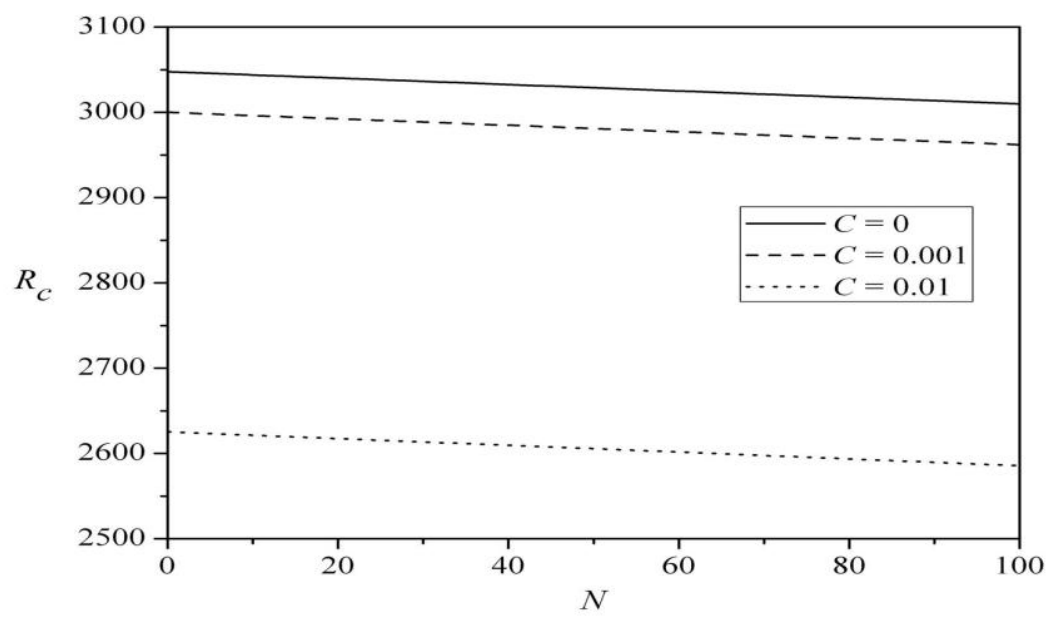

Fig. 2 Variation of $R_{C}$ with $N$ for different values of the Cattaneo number $C$. 


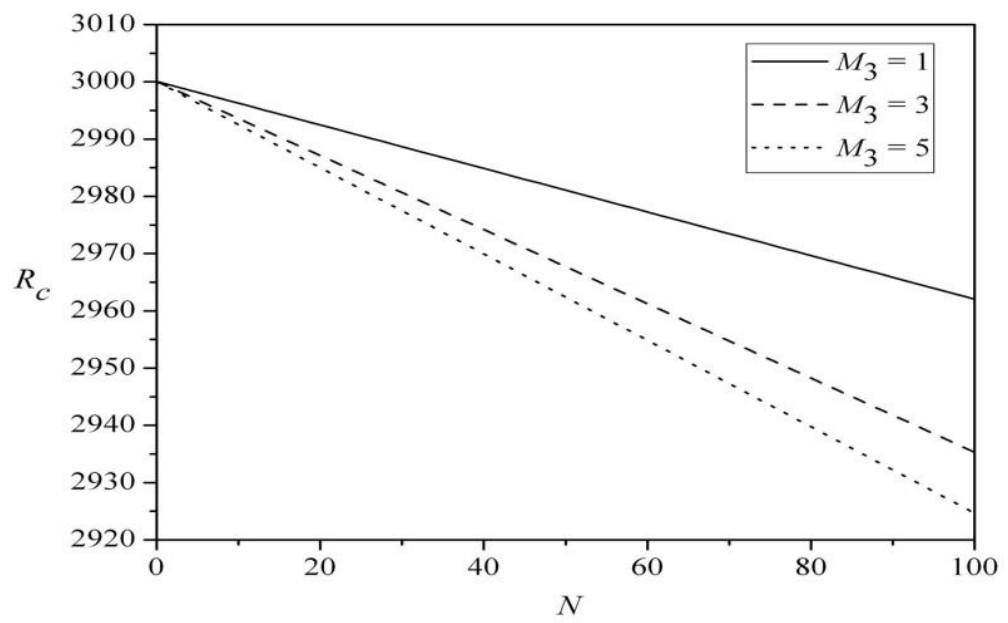

Fig. 3 Variation of $R_{c}$ with $N$ for different values of the magnetization parameter $M_{3}$.

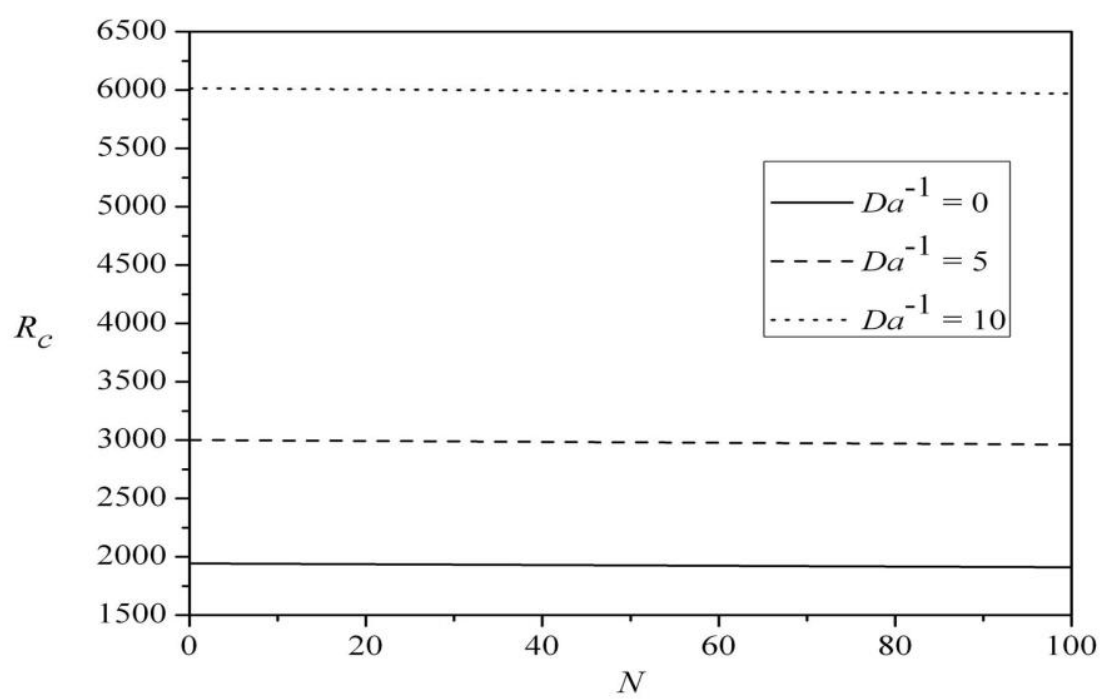

Fig. 4 Variation of $R_{c}$ with $N$ for different values of inverse Darcy number $D a^{-1}$.

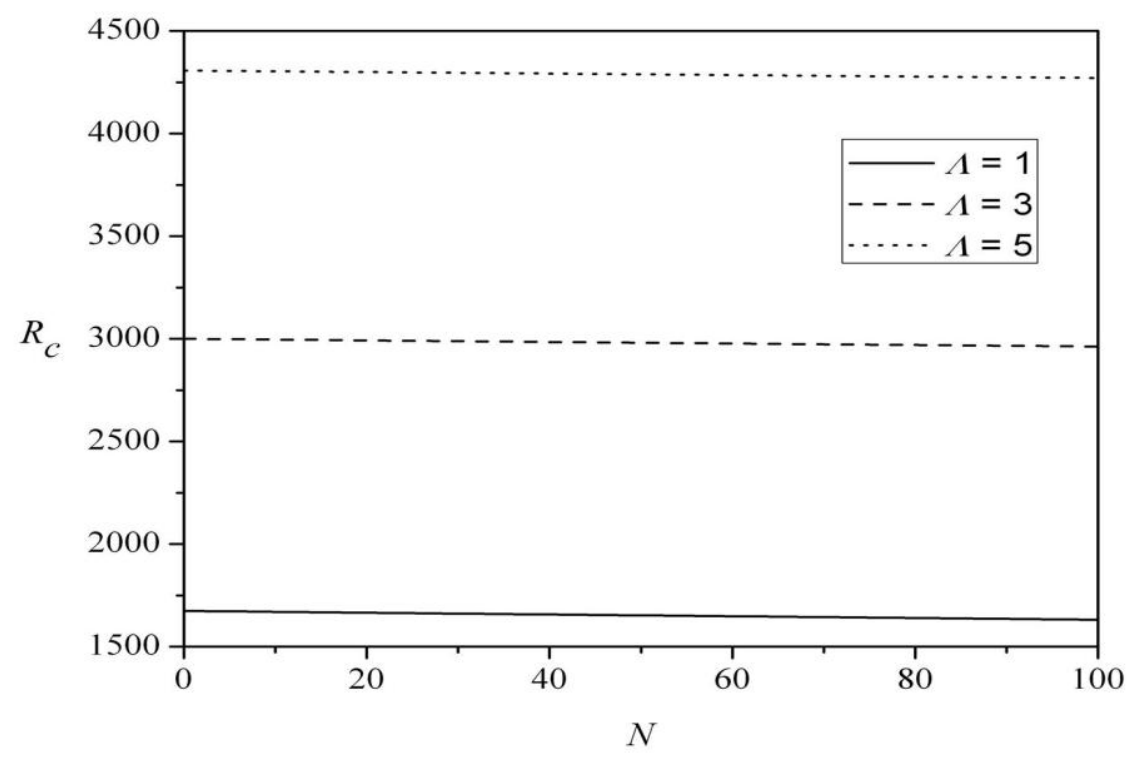

Fig. 5 Variation of $R_{C}$ with $N$ for different values of Brinkman number $\Lambda$. 


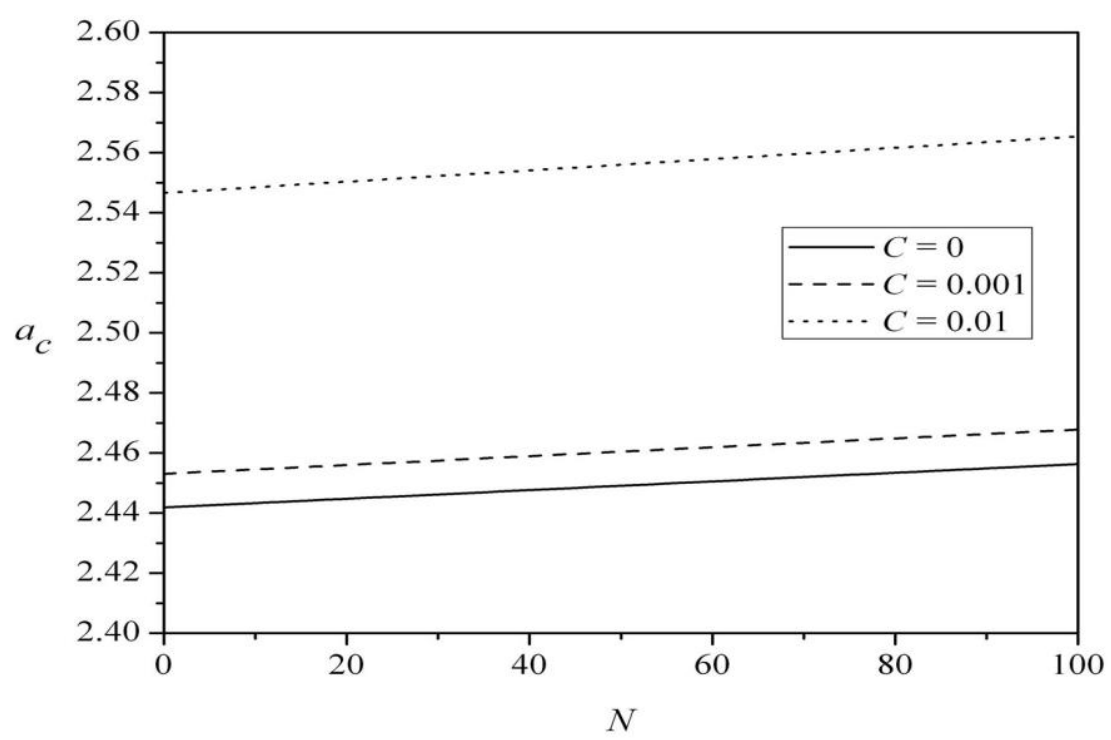

Fig. 6 Variation of $a_{C}$ with $N$ for different values of the Cattaneo number $C$.

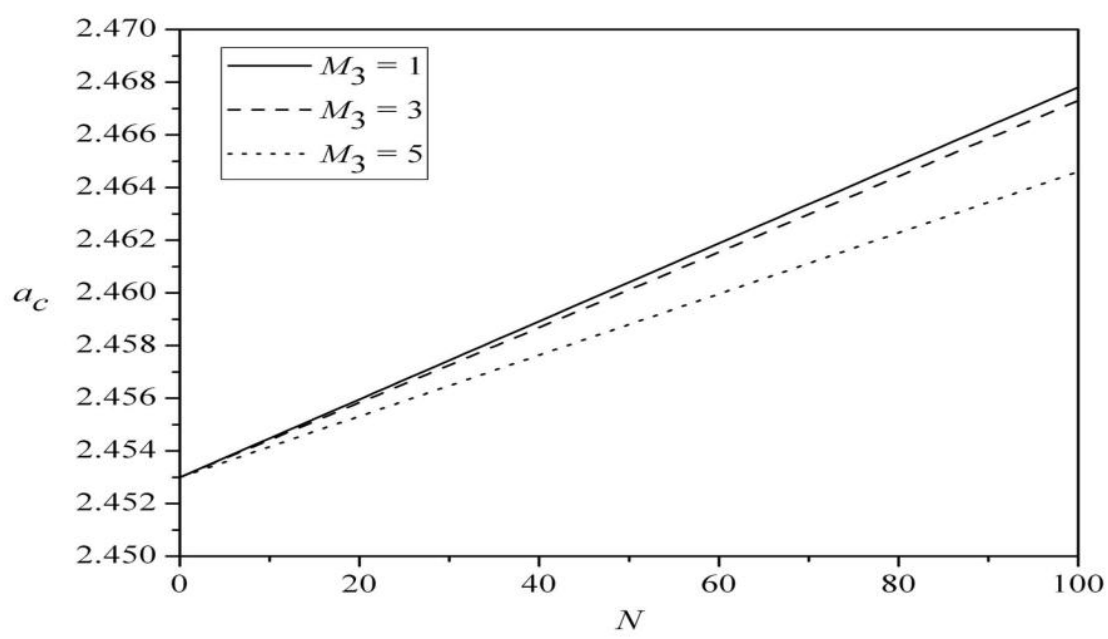

Fig. 7 Variation of $a_{c}$ with $N$ for different values of the magnetization parameter $M_{3}$.

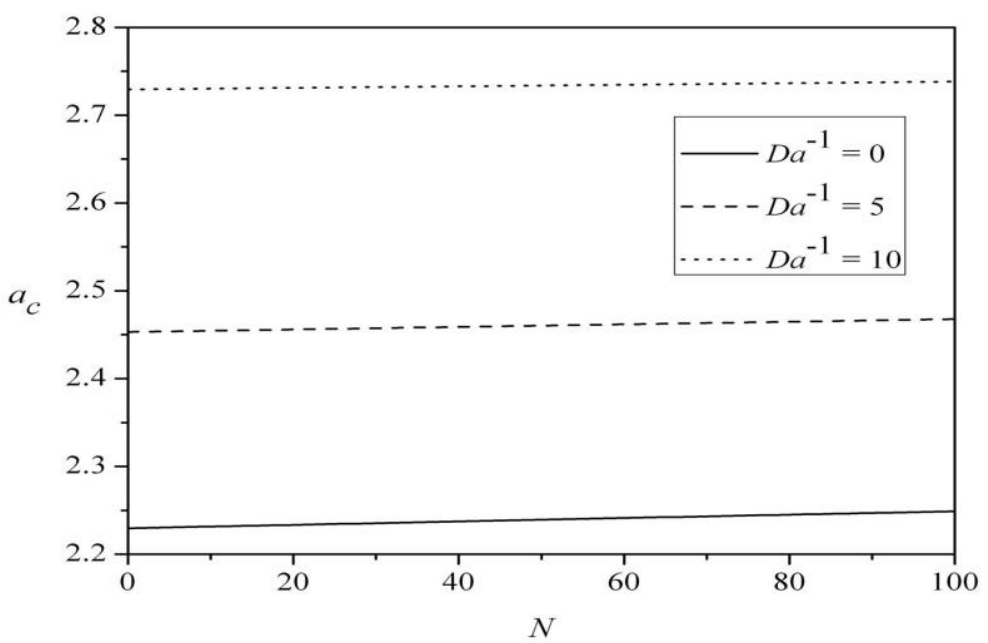

Fig. 8 Variation of $a_{c}$ with $N$ for different values of inverse Darcy number $D a^{-1}$. 


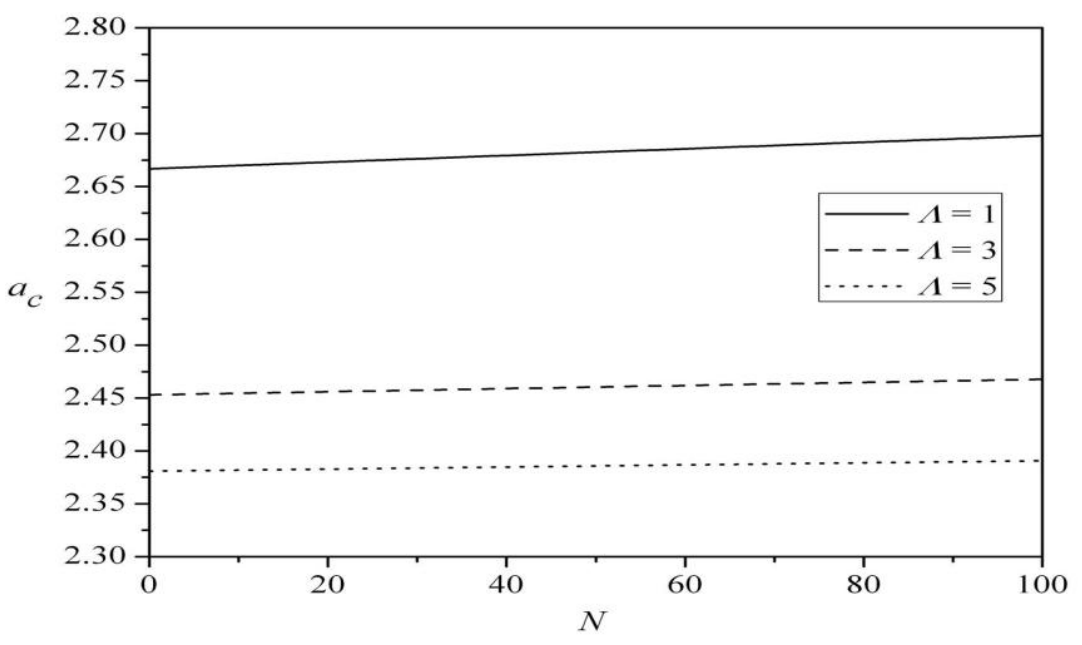

Fig. 9 Variation of $a_{c}$ with $N$ for different values of Brinkman number $\Lambda$.

\section{CONCLUSIONS}

The effect of non-classical heat conduction on the onset of Rayleigh-Bénard instability in a horizontal layer of Darcy-Brinkman porous medium saturated with a Boussinesq-Cattaneo-ferromagnetic liquid subject to the simultaneous action of a vertical magnetic field and a vertical temperature gradient is investigated analytically by the method of small perturbation. A linearized convective instability analysis is performed when both magnetic and buoyancy mechanisms are operative. Inasmuch as the principle of exchange of stabilities is valid for the study at hand, instability criteria are determined in terms of the stationary Rayleigh number $R$, the wavenumber $a$, the Cattaneo number $C$ and magnetic and porous parameters. The following conclusions are drawn:

(i) The Cattaneo heat flux law has a pronounced effect on ferroconvection in a sparsely distributed porous layer.

(ii) The Rayleigh-Bénard problem for a Maxwell-Cattaneo ferromagnetic fluid is always less stable than that with Fourier magnetic fluid.

(iii) In the presence of second sound, the flow is appreciably influenced by magnetic and porous effects.

(iv) The threshold of the stationary instability decreases with increase in the magnetic field strength and the Cattaneo number. Thus the effect of magnetic forces and second sound is to destabilize the system and both cause the convective motion to occur at shorter wavelengths.

(v) There is no appreciable impact of $M_{3}$ on the stability of the system when $N$ is fairly small.

(vi) The stability of the ferromagnetic fluid is reinforced with an increase in the value of the inverse Darcy number and the Brinkman number.

(vii) The critical wavenumber increases with an increase in $N$ and $C$. Thus their effect is to contract the size of convection cells.

(viii) The aspect ratio of convection cell is most sensitive to the effect of porous medium.

\section{Acknowledgements}

The authors are indebted to the Management of Christ University, Bangalore, India for their encouragement and support. The authors are appreciative of the comments of the anonymous reviewers.

\section{References}

[1] V.G. Bashtovoy, B.M. Berkovsky and A.N. Vislovich, Introduction to thermomechanics of magnetic fluids (Washington D.C.: Hemisphere, 1987).

[2] B.A. Finlayson, Convective instability of ferromagnetic fluids, J. Fluid Mech., 40(4), 1970, $753-767$.

[3] M.D. Gupta and A.S. Gupta, Convective instability of a layer of a ferromagnetic fluid rotating about a vertical axis, Int. J. Engg. Sci., 17(3), 1979, 271-277.

[4] K. Gotoh and M. Yamada, Thermal convection in a horizontal layer of magnetic fluids, J. Phys. Soc. Japan, 51(9), 1982, 3042-3048.

[5] E. Blums, Free convection in an isothermic magnetic fluid caused by magnetophoretic transport of particles in the presence of a non-uniform magnetic field, JMMM, 65(2-3), 1987, 343-346.

[6] P.J. Stiles and M. Kagan, Thermoconvective instability of a horizontal layer of ferrofluid in a strong vertical magnetic field, JMMM, 85(1-3), 1990, 196-198. 
[7] S. Odenbach, Convection driven by forced diffusion in magnetic fluids under the influence of strong magnetic field gradients, JMMM, 149(1-2), 1995, 116-118.

[8] S. Aniss, M. Belhaq and M. Souhar, Effects of magnetic modulation on the stability of a magnetic liquid layer heated from above, J. Heat Transfer, 123(3), 2001, 428-433.

[9] A. Abraham, Rayleigh-Bénard convection in a micropolar ferromagnetic fluid, Int. J. Engg. Sci., 40(4), 2002, 449-460.

[10] S. Maruthamanikandan, Effect of radiation on Rayleigh-Bénard convection in ferromagnetic fluids, Int. J. Appl. Mech. Engg., 8(3), 2003, 449-459.

[11] R. Bajaj, Thermodiffusive magnetoconvection in ferrofluids with two-frequency gravity modulation, $J M M M, 288(2)$, 2005, 483-494.

[12] S. Maruthamanikandan, Convective instabilities in Newtonian ferromagnetic, dielectric and other complex liquids, PhD Thesis, Bangalore University, Bangalore, India, 2005.

[13] A. Ramanathan and N. Muchikel, Effect of temperature-dependent viscosity on ferroconvection in a porous medium, Int. J. Appl. Mech. Engg., 11(1), 2006, 93-104.

[14] S. Saravanan, Centrifugal acceleration induced convection in a magnetic fluid saturated anisotropic rotating porous medium, Trans. Porous Media, 77(1), 2009, 79-86.

[15] J. Singh and R. Bajaj, Temperature modulation in ferrofluid convection, Phys. Fluids, 21(6), 2009, p. 064105.

[16] J. Singh and R. Bajaj, Convective instability in a ferrofluid layer with temperature modulated rigid boundaries, Fluid. Dyn. Res., 43(2), 2011, p. 025502.

[17] D.B. Ingham and I. Pop, Transport phenomena in porous media (Oxford: Pergamon, 2005).

[18] D.A. Nield and A. Bejan, Convection in porous media (New York: Springer, 2013).

[19] K. Vafai, Handbook of porous media (New York: Marcel Dekker, 2010).

[20] B. Straughan, Oscillatory convection and the Cattaneo law of heat conduction, Ricerche. Mat., 58, 2009, 157-162.

[21] B. Straughan and F. Franchi, Bénard convection and the Cattaneo law of heat conduction, Proc. Roy. Soc. Edinburgh, 96A, 1984, $175-178$.

[22] G. Lebon and A. Cloot, Bénard-Marangoni instability in a Maxwell-Cattaneo fluid, Phys. Lett., 105A(7), 1984, 361-364.

[23] S.N. Smita and S. Pranesh, Rayleigh-Bénard convection in a second-order fluid with Maxwell-Cattaneo Law, Bull. Soc. Math. Services and Standards, 1(2), 2012, 33-48.

[24] S. Chandrasekhar, Hydrodynamic and hydromagnetic stability (Oxford: Oxford University Press, 1961).

[25] A. Ramanathan, G. Vaidyanathan and S. Maruthamanikandan, The effect of magnetic field dependent viscosity on ferroconvection in a sparsely distributed porous medium, Indian J. Pure Appl. Phys., 40, 2002, 166-171.

[26] B. Straughan, The energy method, stability and nonlinear convection (New York: Springer, 2004). 